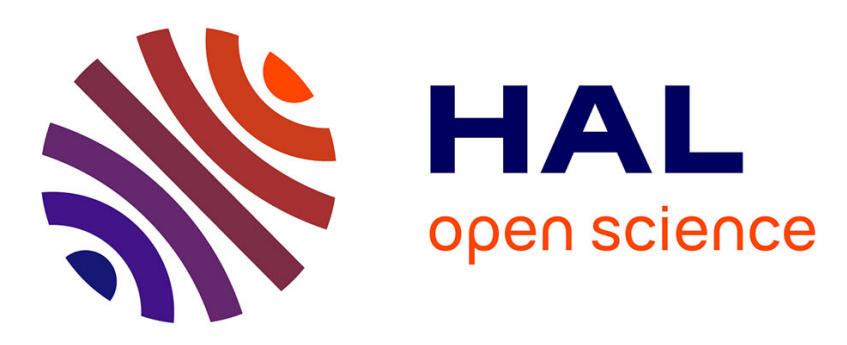

\title{
Precisely positioning the tip of an instrument inserted through an orifice with a free wrist robot: application to prostate biopsies
}

Rémi Chalard, David Reversat, Guillaume G. Morel, Pierre Pierre Mozer, Marie-Aude Vitrani

\section{To cite this version:}

Rémi Chalard, David Reversat, Guillaume G. Morel, Pierre Pierre Mozer, Marie-Aude Vitrani. Precisely positioning the tip of an instrument inserted through an orifice with a free wrist robot: application to prostate biopsies. International Journal of Computer Assisted Radiology and Surgery, 2018, 13 (5), pp.611-618. 10.1007/s11548-018-1718-6 . hal-02049450

\author{
HAL Id: hal-02049450 \\ https://hal.science/hal-02049450
}

Submitted on 6 Mar 2019

HAL is a multi-disciplinary open access archive for the deposit and dissemination of scientific research documents, whether they are published or not. The documents may come from teaching and research institutions in France or abroad, or from public or private research centers.
L'archive ouverte pluridisciplinaire HAL, est destinée au dépôt et à la diffusion de documents scientifiques de niveau recherche, publiés ou non, émanant des établissements d'enseignement et de recherche français ou étrangers, des laboratoires publics ou privés. 


\title{
Precisely positioning the tip of an instrument inserted through an orifice with a free wrist robot.
}

\section{Application to prostate biopsies}

\author{
Rémi Chalard ${ }^{1}$. David Reversat ${ }^{1}$. \\ Guillaume Morel ${ }^{1}$. Pierre Mozer ${ }^{1,2}$. \\ Marie-Aude Vitrani ${ }^{1}$
}

the date of receipt and acceptance should be inserted later

\begin{abstract}
Purpose. Robots with a spherical unactuated wrist are often used for minimally invasive surgery. With such a robot, positioning the wrist center allows to control the instrument tip position when assuming that the insertion site behaves like a lever with a fixed and known fulcrum. In practice, this assumption is not always respected. In this paper we first study the practical consequences of this problem in terms of tip precision positioning. We then propose a solution for robot control that improves the precision compared to the fixed-point assumption approach.

Methods. In the first part of the paper, data recorded during robot-assisted transrectal needle positioning for prostate biopsies ( 9 patients) is exploited to quantify the positioning error induced by the fixed-point model assumption. In the second part of the paper the technique of adaptive control allows to identify on line a model with anisotropy and center displacement. A lab apparatus is used to demonstrate the resulting improvement on tip positioning precision.

Results. Errors obtained by processing the clinical data reach $7.5 \mathrm{~mm}$ at the tip in average. Errors obtained with the lab apparatus drop from $2.4 \mathrm{~mm}$ in average to $0.8 \mathrm{~mm}$ when using adaptive control.
\end{abstract}

Keywords Prostate biopsy · Surgical robotics · keyhole surgery

\section{Introduction}

\subsection{Keyhole surgery}

During keyhole surgery, instrument and/or imaging devices are inserted into a patient through a cannula (trocar) or a natural orifice. When the instrument is manipulated by a robot, respecting the kinematic constraint imposed by the keyhole configuration is an issue. Among the numerous solutions provided in the

\footnotetext{
${ }^{1}$ Institut des Systmes Intelligents et Robotique (ISIR), Sorbonne Université, INSERM U1150, CNRS UMR 7222, F-75005, Paris, France

2 AP-HP, Hôpital de la Pitie Salpetriere, Service d'Urologie, F-75013 Paris, France
} 
literature (mechanical-based strategies such as remote center of motion [2], control law-based strategies with 6 active DoFs robot [14],[9] etc.), anthropomorphic robots possessing 3 actuated joint serially connected to a free-wrist (i.e. a spherical wrist without actuators, [4],[3]) is of great interest. With these devices, as the robot lets the instrument freely orient around the wrist center $W$, the insertion point constraint is automatically respected when the instrument tip is inserted into the patient. This exhibits numerous advantages:

- the robot has less active DoFs then it is smaller, lighter and cheaper than a 6 active DoFs robot;

- its installation is easier as no registration nor precise base positioning is required;

- the wrench applied to the patient at the insertion point is naturally minimized.

A main drawback occurs when a precise location is to be reach by the instrument tip. In such a situation, the robot positions its wrist center $W$ in order to manipulate the tool from outside the patient. Obviously, the position of the tool tip $T$ inside the patient results not only from the position of point $W$ but also from the location of the so-called insertion point. In practice, one can rarely rely on the definition of a fixed insertion point, as backlash or deformation of the tissues surrounding the insertion area occur. This is particularly true for the prostate biopsy application targeted in our research.

\subsection{Prostate targeted biopsies}

A biopsy session is the only exam that allows urologists to diagnose prostate cancer. Although there are different clinical routines to perform this gesture [6] transrectal sampling is by far the most used approach. This method involves sampling of the gland with a needle inserted through a needle-guide attached to an endorectal ultrasound probe. In general, twelve systematic biopsies are distributed in prostate volume [11]. However, in some cases, additional targeted samples may be taken in a given area of interest. Typically, this area corresponds to a region detected earlier on an MRI image or in a previous biopsy session. This surgical gesture is difficult to achieve because prostate experiences large motion and deformation [8]. Meanwhile, precisely positioning the needle constitutes a major medical issue for the prostate biopsy procedure. It explains why many research teams are developing robotic and image guided tools to assist the procedure. An overview of the robot developed for prostate biopsies can be found in [13]. Most of these devices change the clinical procedure (e.g. by using MRI instead of ultrasound imaging [10],[18], transperineal access instead of transrectal access [12],[1] etc.) As for the robots that use endorectal access and ultrasound imaging, it is interesting to note that different solutions are implemented to cope with the kinematic constraint due to the probe insertion through the anus. Most of them assume that the anus plays the role of a 2-DoF kinematic constraint. Examples include the fully actuated robot described in [5] or the robot with remote center of motion used in [7]. On the contrary, our robot Apollo,Fig. 1, fits in the category of free-wrist robots [13] having thus all the advantages described in sec. 1.1 but also the drawback for precise positioning of the needle tip.

In particular, the anus is far from being precisely described by a fixed fulcrum model, resulting in a complex relationship between the position of the robot wrist 

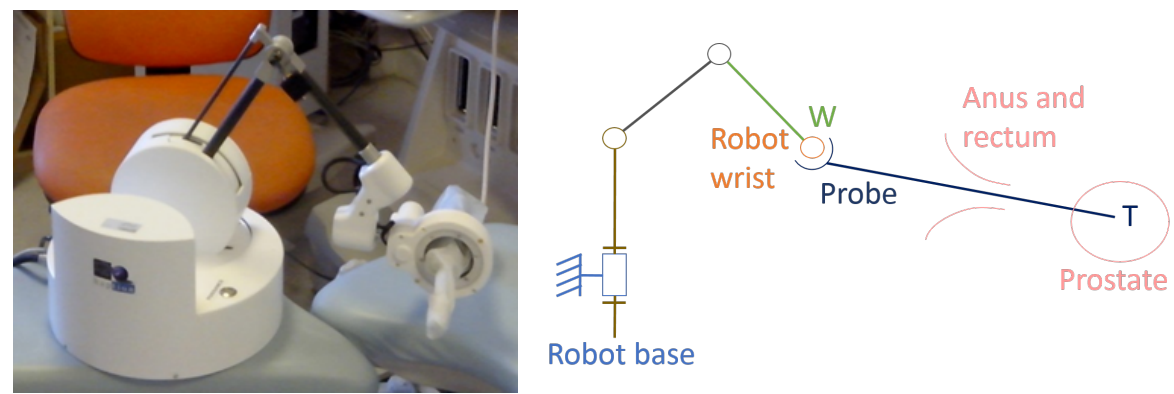

Fig. 1 Apollo robot V1 used during clinical trial and kinematics scheme, [15]

center $W$ and the tip position $T$. This is evidenced in Section 2, where the data recorded during the clinical trial presented in [17] is used to evaluate the positioning error made when assuming that the anus is a fixed point throughout a prostate biopsy procedure. In order to cope with this problem, we propose to modify the robot control law to achieve higher precision, as described section 3 .

\section{Analysis of the tip positioning error when a fixed point hypothesis is made for a prostate biopsy procedure}

A clinical research protocol has been designed as a prospective randomized clinical trial, with respect to the scientific state-of-the-art and the current regulatory frame related to clinical evaluation with non CE marked devices. The sponsor is the Grenoble University Hospital and the clinical trial has been authorized by the relevant french regulatory bodies: Agence Nationale du Médicament et des Produits de Santé (ANSM), the ethical comitee (Comité de Protection des Personnes Sud-Est V, CPP) and the Commission Nationale de l'Informatique et des Libertés (CNIL). The authorization number is NCT02132975.

\subsection{Protocol}

A clinical trial was conduct to evaluate the practical usability and performance of the robot on patients.

Twenty patients (10 with robot and 10 without robot) were included in this proof of concept study. The first intervention with robotic assistance was performed in march 2015. The complete protocol and first results were presented in detail in [17].

For the robotized procedures, the robot is installed in the operating room before the biopsy session starts, see Fig. 2 .

The patient lies on left-lateral decubitus position. The robot is installed on patient's bed, behind his legs and the urologist connects the probe to the robot. An endorectal ultrasound probe fitted with a needle guide is inserted in the patient rectum. A local anesthesia is then preformed. Then the urologist moves the probe toward a first desired biopsy site while the robot is in free mode (allowing the surgeon to freely move the probe). The urologist then switches to the locked mode 


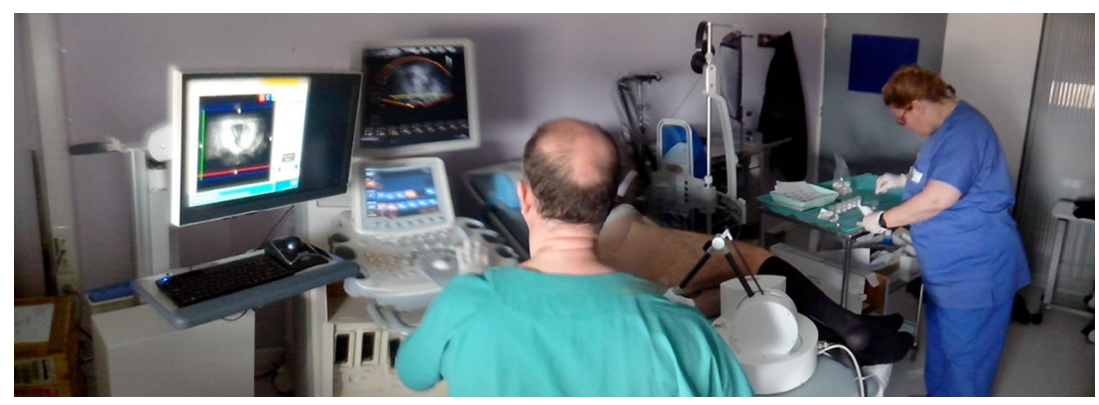

Fig. 2 Installation overview

(allowing to maintain the probe at a chosen location) to take the sample. The urologist had to take 12 samples, according to the conventional sextant scheme (see Fig. 3). The urologist also had to perform 3 supplementary biopsies targeted toward one suspicious lesion observed in MRI data (presenting a suspicious lesion from a preoperative MRI examination was indeed a criterion for inclusion in the clinical trial).

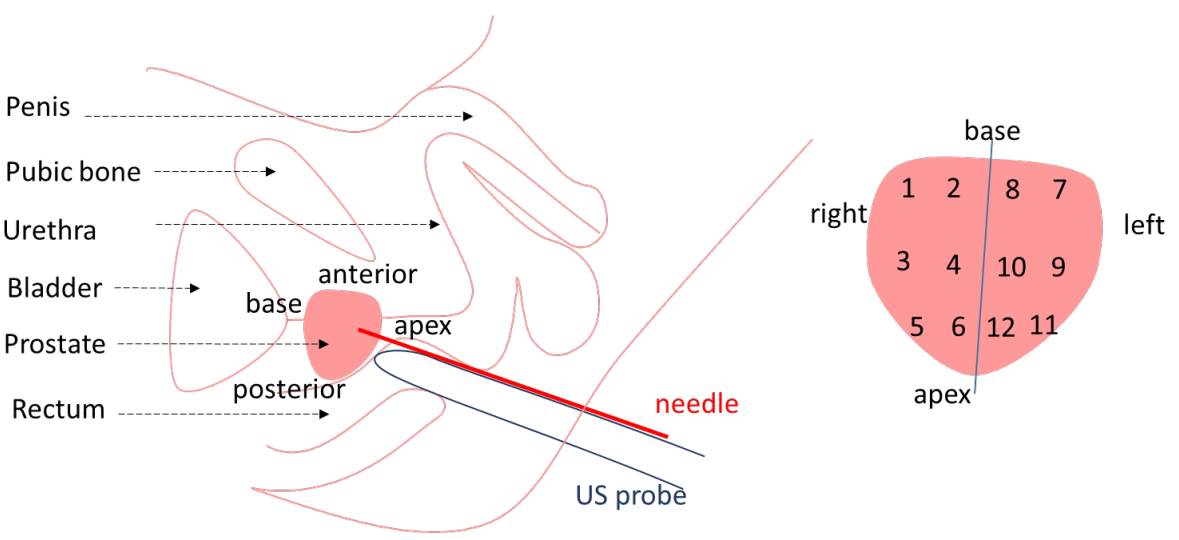

Fig. 3 Conventional sextant scheme

\subsection{Method : Fixed rotation center identification}

During the procedure, both the position of the robot wrist center $W$, which belongs to the probe axis, and the orientation of the probe axis with respect to the robot base frame are recorded. This allows to reconstruct the probe axis in the robot base frame.

From $n$ records of the probe axis localization w.r.t. the robot base frame, one can use a least square algorithm to determine the position of a mean fulcrum position, [16]. This results in an estimated position for a point $A$ around which the probe is supposed to rotate, as illustrated in Fig. 4. 


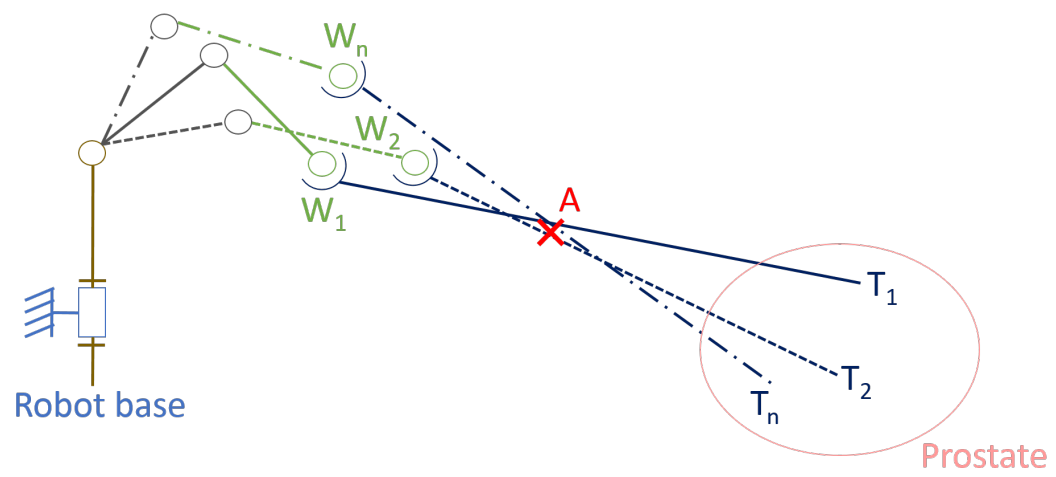

Fig. 4 Identification of fixed point of insertion $A$

In this section, we aim at quantifying the error made in terms of tip positioning if this assumption is made. To this aim we compare two values for the tip position:

$-T_{i}$ is the $i^{\text {th }}$ tip position measured from the 6 robot joint sensors and the robot+probe model;

$-T_{r i}$ is the $i^{t h}$ tip position computed from the $i^{\text {th }}$ position of the wrist center $W_{i}$ and the position of $A$ through which the probe axis is supposed to pass according the fixed point hypothesis under evaluation.

For each sample, the difference between $T_{r i}$ and $T_{i}$ is computed to evaluate the positioning error due to the fixed point hypothesis, as illustrated in Fig. 5:

$$
\varepsilon_{i}=T_{i}-T_{r i}
$$

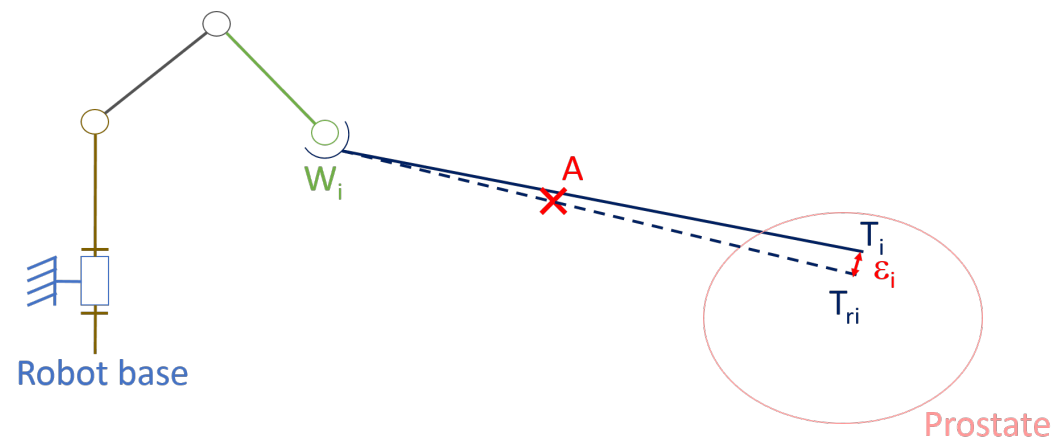

Fig. 5 Positioning error definition

Figure 6 shows the probe axis drawn for $n$ samples recorded during the prostate biopsy procedure for one patient, as well as the point $A$ computed from this sample set. 


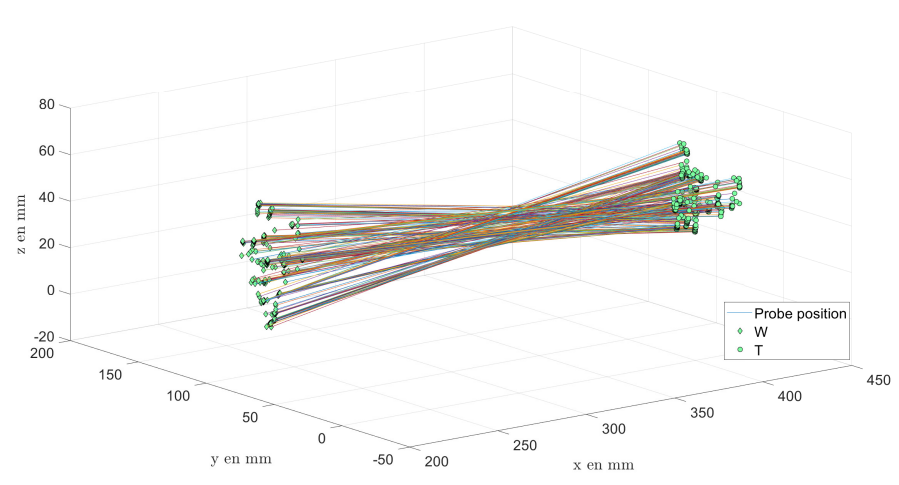

Fig. 6 Typical Data recorded continuously during a session for one patient

\subsection{Results : Positioning error}

In Fig. 6, it is clear that the fixed fulcrum assumption is not valid as not all the probe axes pass through A. Clearly, as the anus and rectum deform during the procedure, the fixed point hypothesis is not valid and leads to large errors in the estimation of the tip position. Results are presented in Fig. 7 for one patient.

The statics of the norm error for each patient are represented in Fig. 8, emphasizing that the average position error generated by the fixed point hypothesis is $7.5 \mathrm{~mm}$, which is large as compared to the size of a prostate (a few centimetres) and to the size of a suspicious lesion (5-10 millimeters).

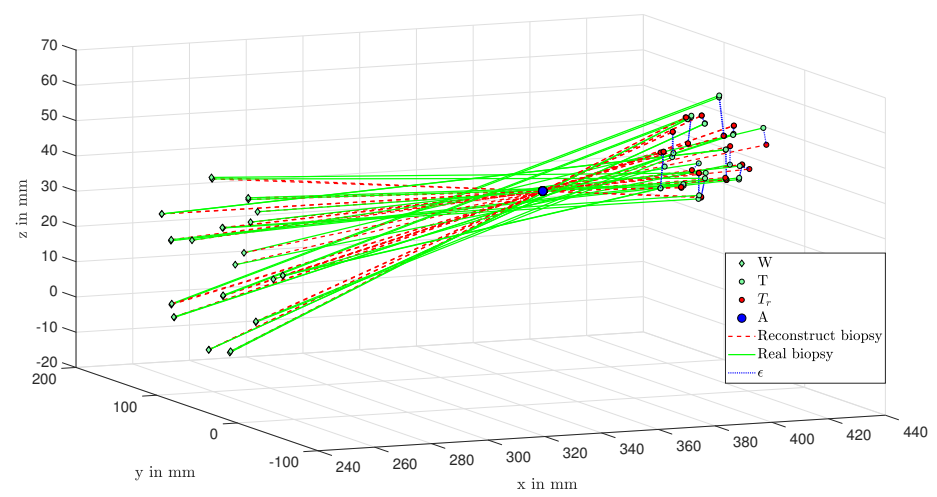

Fig. 7 Positioning error observed at the 12 biopsy configurations during a procedure for one patient

Clearly, the strategy that consists in positioning $T$ by positioning the wrist center position $W$ from a global and constant estimation of $A$ (obtained e.g. through a registration as in [16]) is not possible. Rather, the model that maps $W$ displacements into $T$ displacements is not constant throughout the procedure. In the 


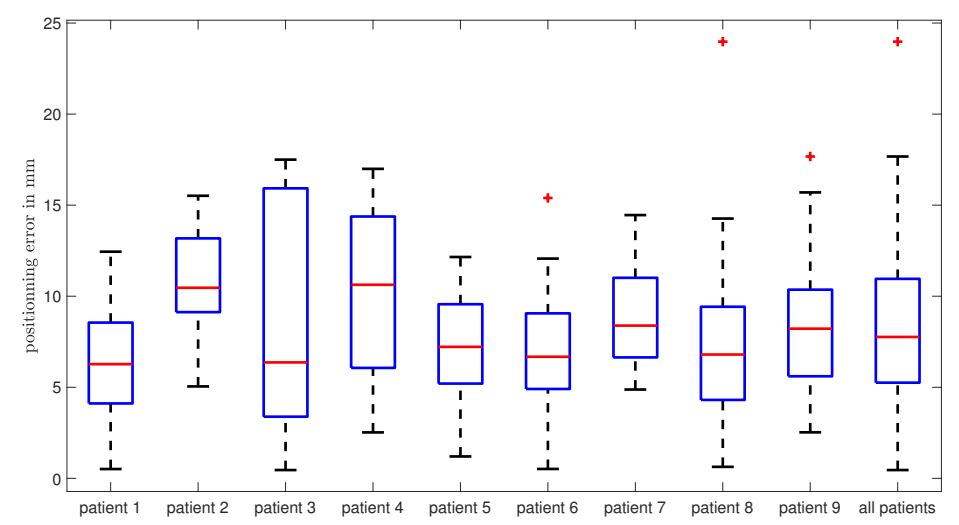

Fig. 8 Statics of the norm error for each patient

next section, we propose to identify this mapping on line at the level of the robot controller.

\section{Adaptive robot control coping with variations of the lever model}

\subsection{Controller}

When a desired location $T_{d}$ is specified for the tip, we want to compute the corresponding desired position of the wrist center, $W_{d}$, which is easily controllable from the robot three first actuated joints. The mapping between $T_{d}$ and $W_{d}$ is not a perfect lever model with a fixed fulcrum, as emphasized in Fig. 7. Clearly, the mapping from $W$ displacements to $T$ displacements depends on how the tissues surrounding the insertion site deform. Considering small movements (local mapping), it is reasonable to assume that this mapping is linear, i.e.:

$$
\delta T=\mathbf{J} \delta W=\left(\begin{array}{ccc}
\mathbf{J}_{x x} & 0 & \mathbf{J}_{x z} \\
0 & 1 & 0 \\
\mathbf{J}_{z x} & 0 & \mathbf{J}_{z z}
\end{array}\right) \delta W .
$$

This particular structure of $\mathbf{J}$ arises from the fact that the instrument inserted is supposed to be rigid and therefore the displacements of $W$ are supposed to be equal to those of $T$ along the penetration axis $y$.

During a manipulation of the instrument, $\mathbf{J}$ has to be permanently updated as it is not constant. An instantaneous estimation of $\mathbf{J}$ can be computed from the instantaneous values of the velocities of $W$ and $T$. Indeed, time differentiation of Eq. (1) leads to:

$$
\mathbf{v}_{T}=\mathbf{J} \mathbf{v}_{W} .
$$

One instantaneous measurement for $\mathbf{v}_{W}$ and $\mathbf{v}_{T}$ is not sufficient to identify the 4 unknown elements of $\mathbf{J}$ as only two equations are available (corresponding to the first and third lines of Eq. (2)). However, exploiting $n$ successive measurements of $\mathbf{v}_{T}$ and $\mathbf{v}_{W}$ while supposing that they were recorded in configurations that are 
close enough to assume that $\mathbf{J}$ is constant, allows to estimate $\mathbf{J}$ by a least square optimization. Denoting $\hat{\mathbf{J}}_{\text {inst }}$ the resulting instantaneous estimate for $\mathbf{J}$, we can implement the in-line estimation of $\mathbf{J}$ at a given instant $k$ as:

$$
\widehat{\mathbf{J}}_{k}=(1-\lambda) \widehat{\mathbf{J}}_{k-1}+\lambda \hat{\mathbf{J}}_{\text {inst }},
$$

where $\lambda$ is a scalar gain verifying $0<\lambda<1$. In practice $\lambda$ is tuned small enough to filter out the measurement noises and large enough to ensure a satisfactory adaptation rate. Figure 9 shows the resulting controller.

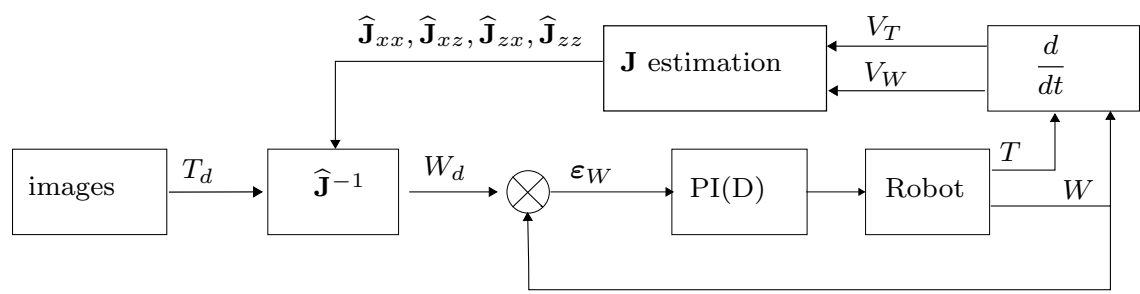

Fig. 9 Control Law

\subsection{Experimental set-up}

The experiments were performed using a new version of Apollo robot which has the same kinematics as those of the first version, described in [13]. The changes arise from stronger actuators and limited cumbersomeness of the wrist. The probe is inserted into 2 devices in order to emulate behaviors that represent what may happen when inserting the probe through both the anus and the rectum. These devices are made with foam more or less tight around the probe thanks to jaws. The apparatus is intentionally built to exhibit anisotropy with a high stiffness along $z$ and a low stiffness along $y$. In this particular configuration, one can assume that $\mathbf{J}_{x z}=\mathbf{J}_{z x} \approx 0$. Therefore only $\mathbf{J}_{x x}$ and $\mathbf{J}_{z z}$ are estimated.
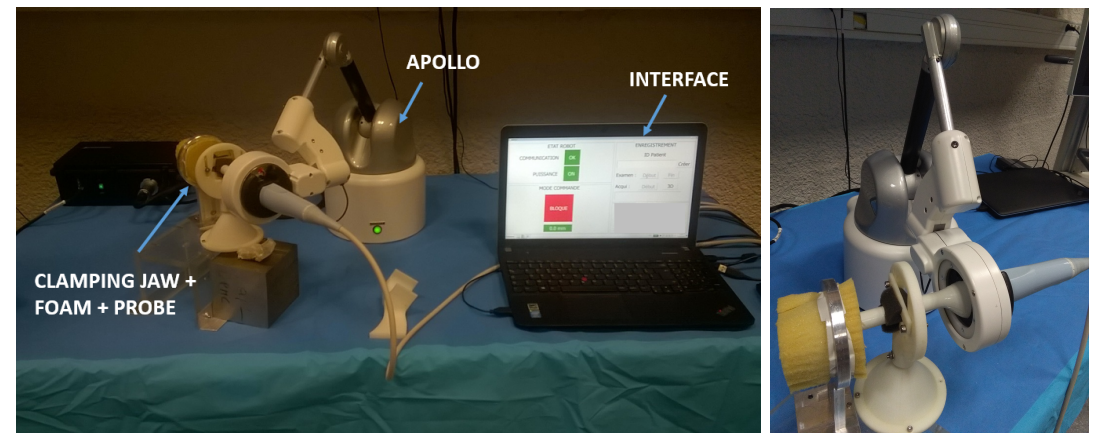

Fig. 10 Experimental set-up

During these experiments, the desired position of the tip $T_{d}$ is computed thanks to the initial tip position (as recorded by the robot) and a desired step. A set of 
$\pm 10 \mathrm{~mm}$ desired steps are sent to the robot to displace the probe tip $T$ along a cross whose dimensions are $10 \mathrm{~mm} \times 10 \mathrm{~mm}$, see Fig. 13. Namely, the $T$ desired trajectory is composed of one step along $x$ followed by two steps along $-x$, and one step along $x$. Then the same sequence is used for the $z$ direction.

The same cross trajectory experiment is performed with:

1. a constant estimate for $\mathbf{J}$ (initial guess corresponding to a lever model computed from a measurement of the fulcrum location);

2. a constantly updated estimation for $\widehat{\mathbf{J}}$.

We then compare the precision at steady state after each step with the two approaches.

\subsection{Results}

Figure 11 (respectively Fig. 12) represents the time evolution of the tip displacement along $x$-axis (resp. $z$-axis) and the time evolution of $\mathbf{J}_{x x}$ (resp. $\mathbf{J}_{z z}$ ) when a step desired displacement is sent along $x$-axis (resp. $z$-axis). On these example trajectories, less error when the on line estimation allows adjusting $\mathbf{J}$.
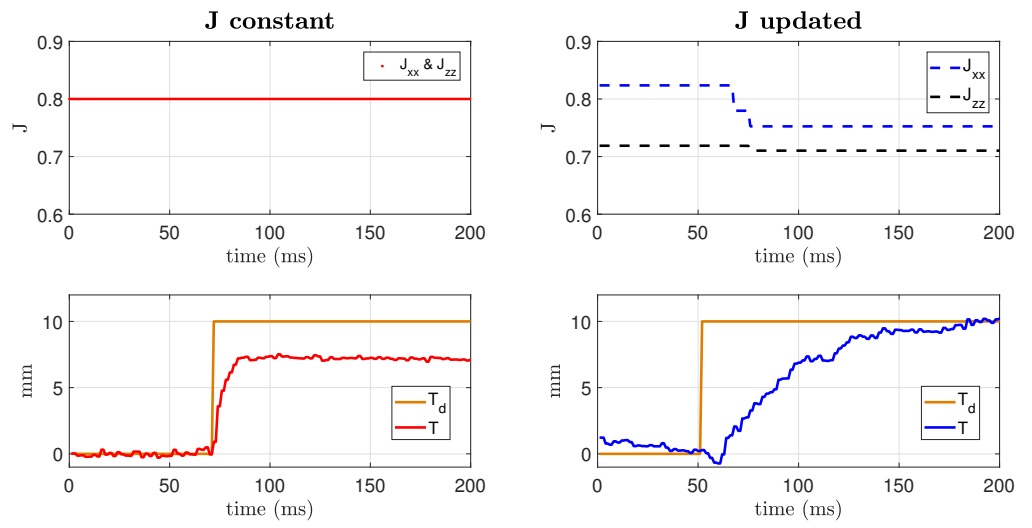

Fig. 11 Tip displacement along $\mathrm{x}$-axis

Further, Fig. 13 shows one example of the recorded trajectories at points $T$ and $P$ during one experiment. It can be seen that the black lines joining $T$ and $P$, which corresponds to the central axis of the probe, do not intersect at the same fixed point $A$. This emphasizes the fact that the experimental setup exhibits a behavior with properties that are comparable to those of the clinical experiments.

In Table 1 we compare the errors obtained when using a constant $\mathbf{J}$ and an updated $\mathbf{J}$. The error computed for each of the 8 steps is the absolute value of the difference between $10 \mathrm{~mm}$ (desired displacement) and the actual displacement measured at point $T$. The mean error when using a constant $\mathbf{J}$ is $2.36 \mathrm{~mm}$ while it is reduced to $0.81 \mathrm{~mm}$ with the adaptive controller.

Clearly, accounting for an anisotropic model and identifying the model in-line reduces the error sgnificantly. 

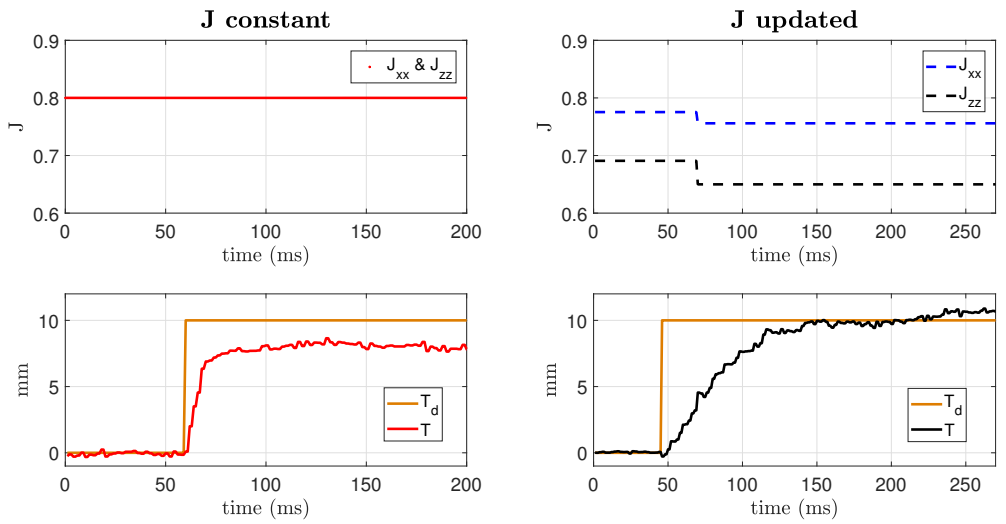

Fig. 12 Tip displacement along z-axis

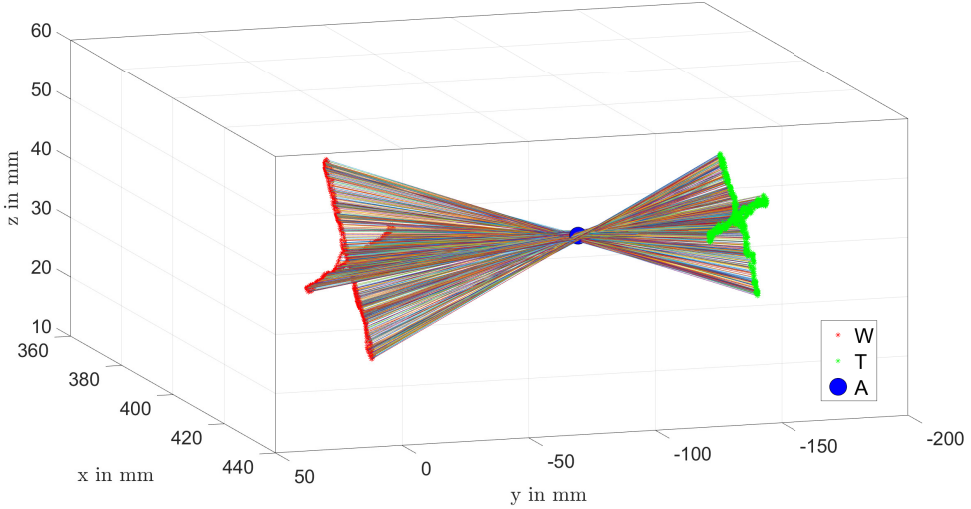

Fig. 13 Displacements of the probe tip along a cross

Table 1 displacement norm for each cross side given by APOLLO with $\mathbf{J}$ constant and updated.

\begin{tabular}{c|c||c}
\hline $10 \mathrm{~mm}$ desired step & with J constant & with $\mathbf{J}$ updated \\
\hline & 7.5 & 12.2 \\
T displacement & 6.4 & 10.5 \\
along x & 8.5 & 10.8 \\
(mm) & 8.1 & 10.3 \\
\hline & 5.7 & 11.1 \\
T displacement & 6.1 & 10.2 \\
along z & 9.8 & 9.8 \\
(mm) & 9 & 8.8 \\
\hline Mean error & $2.36(23.6 \%)$ & $0.81(8.1 \%)$
\end{tabular}




\section{Conclusion}

This paper focuses on an adaptive control law to enhance the instrument tip positioning when using a free wrist robot. In the first part of the paper, data recorded during robot-assisted transrectal needle positioning for prostate biopsies (9 patients) is exploited to quantify the positioning error induced by the fixed-point model assumption. In the second part of the paper the technique of adaptive control allows to identify on line a model with anisotropy and center displacement. A lab apparatus is used to demonstrate the resulting improvement on tip positioning precision. Errors obtained by processing the clinical data reach $7.5 \mathrm{~mm}$ at the tip in average. Errors obtained with the lab apparatus drop from $2.4 \mathrm{~mm}$ in average to $0.8 \mathrm{~mm}$ when using adaptive control. Further experiments need to be done to evaluate this control law under more realistic conditions. In particular, the particular set-up used here allows to assumes that the matrix $\mathbf{J}$ relying tip and wrist displacements is diagonal which is not necessarily the case in clinical context.

Acknowledgements This work has been partially supported by the French state funds managed by the BPI within the FUI'17-MIRAS project and through the funds managed by the ANR within the Investissements d'Avenir programme (Labex CAMI) under reference ANR11-LABX-0004.

\section{References}

[1] H. Bassan et al. "A Novel Manipulator for 3D Ultrasound Guided Percutaneous Needle Insertion". In: Robotics and Automation, 2007 IEEE International Conference on. April 2007, pp. 617-622.

[2] G. Guthart and J. Salisbury. "The Intuitive Telesurgery System : Overview and Application". In: Proc IEEE International Conference on Robotics and Automation, 2000 (2000), pp. 618-621.

[3] T. Ortmaier and G. Hirzinger. "Cartesian control issues for minimally invasive robot surgery". In: Proceedings. 2000 IEEE/RSJ International Conference on Intelligent Robots and Systems (IROS 2000) (Cat. No.00CH37113). Vol. 1. 2000, 565-571 vol.1. DOI: 10.1109/IROS.2000.894664.

[4] S. C. Low and L. Phee. "A review of master-slave robotic systems for surgery". In: IEEE Conference on Robotics, Automation and Mechatronics, 2004. Vol. 1. Dec. 2004, 37-42 vol.1. DOI: 10.1109/RAMECH. 2004.1438888.

[5] C. M. Schneider, A. M. Okamura, and G. Fichtinger. "A robotic system for transrectal needle insertion into the prostate with integrated ultrasound". In: 2004 IEEE International Conference on Robotics and Automation, 2004. Proceedings. ICRA '04. Vol. 1. Apr. 2004, 365-370 Vol.1. DOI: 10.1109/ROBOT. 2004.1307177.

[6] A. Villers et al. "Conditions de réalisation et schéma de ponctions lors d' une première série de biopsies prostatiques." In: Progrès en Urologie 14 (2004), pp. 144-153.

[7] Z. Wei et al. "3D TRUS Guided Robot Assisted Prostate Brachytherapy". In: Medical Image Computing and Computer-Assisted Intervention, MICCAI 2005. Ed. by JamesS. Duncan and Guido Gerig. Vol. 3750. Lecture Notes in Com- 
puter Science. Springer Berlin Heidelberg, 2005, pp. 17-24. ISBN: 978-3-54029326-2.

[8] M. Marchal. "Modélisation des tissus mous dans leur environnement pour 1 xfffdfffdfffd aide aux gestes médico-chirurgicaux". In: PhD thesis from Joseph Fourier University (2006).

[9] R. Konietschke et al. "The DLR MiroSurge - A Robotic System for Surgery". In: IEEE International Conference on Robotics and Automation. 2009, pp. 1589 1590 .

[10] A. Krieger et al. "An MRI-Compatible Robotic System With Hybrid Tracking for MRI-Guided Prostate Intervention". In: IEEE Transactions on Biomedical Engineering 58.11 (Nov. 2011), pp. 3049-3060. ISSN: 0018-9294. DOI: 10. 1109/TBME. 2011.2134096.

[11] A. Ouzzane et al. "Recommandations pour la bonne pratique des biopsies prostatiques". In: Progrès en Urologie 21.1 (Jan. 2011), pp. 18-28. ISSN: 11667087. DOI: $10.1016 / \mathrm{j}$.purol .2010 .07 .001$.

[12] N. Hungr et al. "A 3D Ultrasound Robotic Prostate Brachytherapy System with Prostate Motion Tracking". In: IEEE Transactions on Robotics 28.6 (Dec. 2012), pp. 1382-1397. DOI: 10.1109/TRO.2012.2203051.

[13] C. Poquet et al. "A novel comanipulation device for assisting needle placement in ultrasound guided prostate biopsies". In: IEEE, Nov. 2013, pp. 40844091. DOI: 10.1109/IROS.2013.6696941.

[14] C. D. Pham et al. "Analysis of a moving remote center of motion for roboticsassisted minimally invasive surgery". In: 2015 IEEE/RSJ International Conference on Intelligent Robots and Systems (IROS). Sept. 2015, pp. 1440-1446. DOI: 10.1109/IROS.2015.7353557.

[15] C. Poquet et al. "An Endorectal Ultrasound Probe Comanipulator With Hybrid Actuation Combining Brakes and Motors". In: IEEE/ASME Transactions on Mechatronics 20.1 (Feb. 2015), pp. 186-196. ISSN: 1083-4435, 1941014X. DOI: 10.1109/TMECH. 2014.2314859.

[16] L. Dong and G. Morel. "Robust trocar detection and localization during robot-assisted endoscopic surgery". In: 2016 IEEE International Conference on Robotics and Automation (ICRA). May 2016, pp. 4109-4114. DOI: 10.1109/ ICRA . 2016.7487602.

[17] M.-A. Vitrani et al. "Prostate biopsies assisted by comanipulated probeholder : First in Man". In: International Journal of Computer Assisted Radiology and Surgery Springer Berlin Heidelberg 11.6 (June 2016), 1153-1161 "Bench to Bedside Award". DOI: 10.1007/s11548-016-1399-y.

[18] D. Stoianovici et al. "MR Safe Robot, FDA Clearance, Safety and Feasibility of Prostate Biopsy Clinical Trial". In: IEEE/ASME Transactions on Mechatronics 22.1 (Feb. 2017), pp. 115-126. ISSN: 1083-4435, 1941-014X. DOI: 10.1109/TMECH. 2016.2618362. 